\title{
Conditions for separability in generalized Laplacian matrices and nonnegative matrices as density matrices
}

\author{
Chai Wah Wu \\ IBM Research Division, Thomas J. Watson Research Center \\ P. O. Box 218, Yorktown Heights, NY 10598, U. S. A.
}

\begin{abstract}
Recently, Laplacian matrices of graphs are studied as density matrices in quantum mechanics. We continue this study and give conditions for separability of generalized Laplacian matrices of weighted graphs with unit trace. In particular, we show that the Peres-Horodecki positive partial transpose separability condition is necessary and sufficient for separability in $\mathbb{C}^{2} \otimes \mathbb{C}^{q}$. In addition, we present a sufficient condition for separability of generalized Laplacian matrices and diagonally dominant nonnegative matrices.
\end{abstract}

Key words: density matrix, entanglement, graph theory, Laplacian matrix, nonnegative matrix, partial transpose.

PACS: 02.10.Ox, 02.10.Ud, 02.10.Yn, 03.65.-w, 03.65.Ud.

\section{Introduction}

Due to novel applications of quantum mechanics in recent years such as quantum teleportation, quantum cryptography and quantum computing [1], there is much recent interest in studying entanglement in quantum systems. One important problem is to determine whether a given state operator is entangled or not. This is especially difficult for mixed state operators. In Ref. [2], normalized Laplacian matrices of graphs are considered as density matrices, and their entanglement properties are studied. The reason for studying this subclass of density matrices is that simpler and stronger conditions for entanglements can be found. In Ref. [3] a conjecture was proposed on a necessary

Email address: cwwu@us.ibm.com, chaiwahwu@member.ams.org (Chai Wah $\mathrm{Wu})$. 
and sufficient condition for separability of such density matrices and the conjecture was verified for some special classes of graphs.

The purpose of this paper is to further this study and give some generalizations of these results. In particular, we show that the Peres-Horodecki positive partial transpose condition is necessary and sufficient for Laplacian matrices of weighted graphs to be separable in $\mathbb{C}^{2} \otimes \mathbb{C}^{q}$. Furthermore, we give a sufficient condition for Laplacian matrices and diagonally dominant nonnegative matrices to be separable in $\mathbb{C}^{p} \otimes \mathbb{C}^{q}$.

\section{Density matrices, separability, and partial transpose}

We use $I$ and $\mathbf{0}$ to denote the identity matrix and the zero matrix respectively. A state of a finite dimensional quantum mechanical system is described by a state operator or a density matrix $\rho$ acting on $\mathbb{C}^{n}$ which is Hermitian and positive semidefinite with unit trace. A state operator is called a pure state if it has rank one. Otherwise the state operator is mixed. An $n$ by $n$ density matrix $\rho$ is separable in $\mathbb{C}^{p} \otimes \mathbb{C}^{q}$ with $n=p q$ if it can be written as $\sum_{i} c_{i} \rho_{i} \otimes \eta_{i}$ where $\rho_{i}$ are $p$ by $p$ density matrices and $\eta_{i}$ are $q$ by $q$ density matrices with $\sum_{i} c_{i}=1$ and $c_{i} \geq 0 .{ }^{1} \mathrm{~A}$ density matrix that is not separable is called entangled. Entangled states are necessary to invoke behavior that can not be explained using classical physics and enable applications such as quantum teleportation and quantum cryptography.

We denote the $(i, j)$-th element of a matrix $A$ as $A_{i j}$. Let $f$ be the canonical bijection between $\{1, \ldots, p\} \times\{1, \ldots, q\}$ and $\{1, \ldots, p q\}: f(i, j)=(i-1) q+j$. For a $p q$ by $p q$ matrix $A$, if $f(i, j)=k$ and $f\left(i_{2}, j_{2}\right)=l$, we will sometimes write $A_{k l}$ as $A_{(i, j)\left(i_{2}, j_{2}\right)}$.

Definition 1 The $(p, q)$-partial transpose $A^{p T}$ of an $n$ by $n$ matrix $A$, where $n=p q$, is given by:

$$
A_{(i, j)(k, l)}^{p T}=A_{(i, l)(k, j)}
$$

We will usually remove the prefix " $(p, q)$ " if $p$ and $q$ are clear from context. In matrix form, the partial transpose is constructed as follows. If $A$ is decomposed

$\overline{1}$ This definition can be extended to composite systems of multiple states, but here we only consider decomposition into the tensor product of two component states. 
into $p^{2}$ blocks:

$$
A=\left(\begin{array}{cccc}
A^{1,1} & A^{1,2} & \cdots & A^{1, p} \\
A^{2,1} & A^{2,2} & \cdots & A^{2, p} \\
\vdots & \vdots & & \vdots \\
A^{p, 1} & A^{p, 2} & \cdots & A^{p, p}
\end{array}\right)
$$

where each $A^{i, j}$ is a $q$ by $q$ matrix, then $A^{p T}$ is given by:

$$
A^{p T}=\left(\begin{array}{cccc}
\left(A^{1,1}\right)^{T} & \left(A^{1,2}\right)^{T} & \cdots & \left(A^{1, p}\right)^{T} \\
\left(A^{2,1}\right)^{T} & \left(A^{2,2}\right)^{T} & \cdots & \left(A^{2, p}\right)^{T} \\
\vdots & \vdots & & \vdots \\
\left(A^{p, 1}\right)^{T} & \left(A^{p, 2}\right)^{T} & \cdots & \left(A^{p, p}\right)^{T}
\end{array}\right)
$$

It is clear that if $A$ is Hermitian, then so is $A^{p T}$. Peres [4] introduced the following necessary condition for separability:

Theorem 1 If a density matrix $\rho$ is separable, then $\rho^{p T}$ is positive semidefinite, i.e. $\rho^{p T}$ is a density matrix.

Horodecki et al. [5] showed that this condition is sufficient for separability in $\mathbb{C}^{2} \otimes \mathbb{C}^{2}$ and $\mathbb{C}^{2} \otimes \mathbb{C}^{3}$, but not for other tensor products. A density matrix having a positive semidefinite partial transpose is often referred to as the Peres-Horodecki condition for separability.

\section{Laplacian matrices of graphs as density matrices}

For a graph $\mathcal{G}$, the Laplacian matrix $L(\mathcal{G})$ is defined as $D-A$ where $D$ is the diagonal matrix of vertex degrees and $A$ is the adjacency matrix. The Laplacian matrix is symmetric positive semidefinite, has zero row sums and has a simple zero eigenvalue if and only if the graph is connected [6]. Let $|E|$ be the total number of edges in the graph. Then $\frac{1}{2|E|} L(\mathcal{G})$ has unit trace and is thus a density matrix. In Ref. [2] properties of such density matrices are obtained by studying the properties of the underlying graph. If $A$ is the adjacency matrix of $\mathcal{G}$, let $\mathcal{G}^{p T}$ be the graph with adjacency matrix $A^{p T}$. Graphically, $\mathcal{G}^{p T}$ is obtained from $\mathcal{G}$ as follows. Let the vertex $k=f(i, j)$ be located at coordinate $(i, j)$. Then $\mathcal{G}^{p T}$ is obtained from $\mathcal{G}$ by reflecting each edge vertically (or horizontally) around the midpoint of the edge. Fig. 1 shows an example for $p=3, q=4$. 


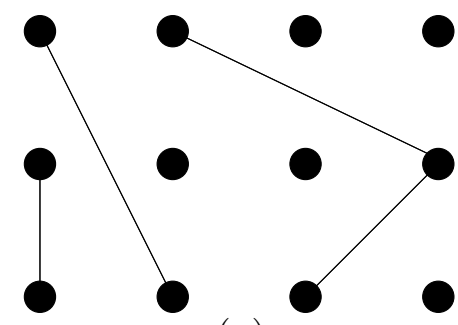

(a)

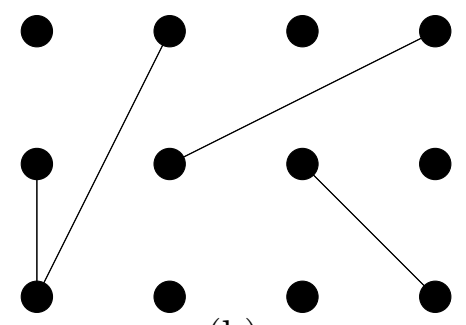

(b)

Fig. 1. (a) A graph $\mathcal{G}$ and (b) its partial transpose $\mathcal{G}^{p T}$ for the case $p=3, q=4$.

In Ref. [3] it was shown that the vertex degrees of $\mathcal{G}$ equaling the vertex degrees of $\mathcal{G}^{p T}$ is a necessary condition for separability and it was conjectured that this is also a sufficient condition. The sufficiency is shown for perfect matchings in $\mathbb{C}^{2} \otimes \mathbb{C}^{q}$ and nearest point graphs.

\section{Generalized Laplacian matrices of weighted graphs}

Let $S$ be the set of symmetric real matrices with nonnegative row sums and nonpositive off-diagonal elements. Then $S$ is a subset of generalized Laplacian matrices as defined in Ref. [6]. We can associate a simple weighted graph to a matrix $A \in S$ : for $i \neq j, A_{i j} \neq 0$ corresponds to an edge from vertex $i$ to vertex $j$ with weight $-A_{i j}>0$. Matrices in $S$ are positive semidefinite. If the corresponding graph is connected, then the smallest eigenvalue is simple. Let $S_{1}$ be the matrices in $S$ with unit trace and let $S_{1}^{0}$ be matrices in $S_{1}$ with zero row sums. We focus on $S_{1}$ and $S_{1}^{0}$ which are sets of density matrices. We start with the following necessary condition for separability:

Theorem 2 Let $A$ be a density matrix with zero row sums. If $A^{p T}$ does not have zero row sums, then $A$ is not separable.

Proof: Let $e=(1, \ldots 1)^{T}$. Then $0=e^{T} A e=\sum_{i j} A_{i j}=\sum_{i j} A_{i j}^{p T}=e^{T} A^{p T} e$. Since $A^{p T} e \neq 0$, if $e$ is an eigenvector of $A^{p T}$, it means that it corresponds to a nonzero eigenvalue of $A^{p T}$ which contradicts the fact that $e^{T} A^{p T} e=0$. Therefore $e=\sum_{i} a_{i} v_{i}$ is a linear combination of several eigenvectors $v_{i}$ of $A^{p T}$, one of which is not in the kernel of $A^{p T}$. Since $0=e^{T} A^{p T} e=\sum_{i} \lambda_{i}\left|a_{i}\right|^{2}\left\|v_{i}\right\|^{2}$, this means that one of the $\lambda_{i}$ is negative, which implies that $A^{p T}$ is not positive semidefinite, hence by Theorem $1, A$ is not separable.

Note that Theorem 2 is applicable to general density matrices, i.e. not necessarily Laplacian matrices of graphs. The above result generalizes Theorem 1 in Ref. [3] and is weaker than the Peres-Horodecki condition, but is easier to verify when $A$ happens to have zero row sums. On the other hand, for matrices in $S_{1}^{0}$, this condition is equivalent to the Peres-Horodecki condition. 
Theorem 3 Let $A$ be a density matrix in $S_{1}^{0}$. The matrix $A^{p T}$ has zero row sums if and only if $A^{p T}$ is positive semidefinite.

Proof: One direction follows from the proof of Theorem 2. If $A^{p T}$ has zero row sums, it is a matrix in $S_{1}^{0}$ and hence positive semidefinite.

It is easy to show that for $A \in S$ with corresponding graph $\mathcal{G}, A^{p T}$ having the same row sums as $A$ is equivalent to the vertex degrees of $\mathcal{G}$ and $\mathcal{G}^{p T}$ being equal. $^{2}$

For $A \in S$, an edge corresponding to $A_{(i, j)\left(i^{\prime}, j^{\prime}\right)} \neq 0$ is called entangled if $i \neq i^{\prime}$ and $j \neq j^{\prime}$. It's easy to see that if an edge corresponding to $A_{(i, j)\left(i^{\prime}, j^{\prime}\right)}$ is not entangled, then $A_{(i, j)\left(i^{\prime}, j^{\prime}\right)}=A_{(i, j)\left(i^{\prime}, j^{\prime}\right)}^{p T}$. In Ref. [2] it was conjectured that normalized Laplacian matrices of graphs where all entangled edges are adjacent to the same vertex are not separable. By Theorem 2 this conjecture is true. In particular, it is true for the larger set of density matrices in $S_{1}^{0}$ since the degree of this vertex must necessarily decrease in $A^{p T}$.

\section{Sufficient and necessary conditions for separability}

Theorem 4 Let $F$ be a $q$ by q matrix of the form $F=\left(\begin{array}{l}U \\ \mathbf{0}\end{array}\right)$ and $D$ be a $q$ by $q$ diagonal matrix of the form $D=\left(\begin{array}{l}I \\ \mathbf{0}\end{array}\right)$ where $U$ is an unitary matrix of the same size as I. Then the matrix

$$
A=\frac{1}{2 \operatorname{Tr}(D)}\left(\begin{array}{cc}
D & F \\
F^{\dagger} & D
\end{array}\right)
$$

is a density matrix and is separable in $\mathbb{C}^{2} \otimes \mathbb{C}^{q}$.

Proof: Since $U$ is unitary, it can be written as $U=\sum_{i} \lambda_{i} v_{i} v_{i}^{\dagger}$ where $\lambda_{i}$ and $v_{i}$ are the eigenvalues and eigenvectors of $U$ respectively and $v_{i}^{\dagger}$ denotes the complex conjugate transpose of $v_{i}$. Furthermore, all eigenvalues of $U$ are on

2 The vertex degree of $v$ in a weighted graph is the sum of the weights of all the edges connected to $v$. 
the unit circle and written as $\lambda_{i}=e^{\theta_{i}}$ with imaginary numbers $\theta_{i}$. Define

$$
w_{i}=\left(\begin{array}{c}
v_{i} \\
0 \\
\vdots \\
0
\end{array}\right)
$$

This means that $F=\sum_{i} \lambda_{i} w_{i} w_{i}^{\dagger}$. Furthermore, $\sum_{i} v_{i} v_{i}^{\dagger}=I$ implies that $\sum_{i} w_{i} w_{i}^{\dagger}=D$. Define $x_{i}=\left(\begin{array}{c}e^{\frac{\theta_{i}}{2}} \\ e^{\frac{-\theta_{i}}{2}}\end{array}\right) \otimes w_{i}$. Then

$$
\sum_{i} x_{i} x_{i}^{\dagger}=\left(\begin{array}{cc}
\sum_{i} w_{i} w_{i}^{\dagger} & \sum_{i} \lambda_{i} w_{i} w_{i}^{\dagger} \\
\sum_{i} \overline{\lambda_{i}} w_{i} w_{i}^{\dagger} & \sum_{i} w_{i} w_{i}^{\dagger}
\end{array}\right)=\left(\begin{array}{cc}
D & F \\
F^{\dagger} & D
\end{array}\right)
$$

which shows that $A$ is positive semidefinite and is separable.

Definition $2 C$ is a simple circuit matrix if there exists distinct integers $i_{1}, \ldots, i_{k}(k \geq 1)$ such that $C_{i_{m} i_{m+1}}=1$ for $m=1, \ldots, k-1, C_{i_{k} i_{1}}=1$ and $C_{i j}=0$ otherwise.

Corollary 1 If $C$ is a $q$ by $q$ simple circuit matrix and $D$ is the diagonal matrix with the row sums of $C$ on the diagonal, then the matrix

$$
A=\frac{1}{2 \operatorname{Tr}(D)}\left(\begin{array}{cc}
D & -C \\
-C^{T} & D
\end{array}\right)
$$

is a density matrix and is separable in $\mathbb{C}^{2} \otimes \mathbb{C}^{q}$.

Proof: There exists permutation matrices $P, Q$ such that $Q^{T} C Q$ is of the form $\left(\begin{array}{ll}P & \\ & 0\end{array}\right)$. Therefore without loss of generality, we assume that $C$ is of the form $C=\left(\begin{array}{ll}P & \\ & \mathbf{0}\end{array}\right)$. This means that $D=\left(\begin{array}{ll}I & \\ & \mathbf{0}\end{array}\right)$. Since $-P$ is unitary, the result follows from Theorem 4 .

Definition 3 A matrix is line sum symmetric if the $i$-th column sum is equal to the $i$-th row sum for each $i$.

Theorem 5 Let $A$ be a $2 q$ by $2 q$ density matrix in $S_{1}$. If $A^{p T}$ has the same row sums as $A$, then the matrix $A$ is separable in $\mathbb{C}^{2} \otimes \mathbb{C}^{q}$. 
Proof: It is easy to see that $A$ can be decomposed into:

$$
A=\left(\begin{array}{ll}
A_{1} & \\
& 0
\end{array}\right)+\left(\begin{array}{ll}
\mathbf{0} & \\
& A_{2}
\end{array}\right)+\left(\begin{array}{cc}
D_{1} & -B \\
-B^{T} & D_{2}
\end{array}\right)
$$

where $A_{1}$ and $A_{2}$ are symmetric matrices and $D_{1}$ and $D_{2}$ are diagonal matrices with the row sums of $B$ and $B^{T}$ on the diagonal respectively. It is easy to see that $A_{1}$ and $A_{2}$ are in $S$ and thus are positive semidefinite. The first term $\left(\begin{array}{ll}A_{1} & \\ & 0\end{array}\right)$ can be separated as $\left(\begin{array}{ll}1 & 0 \\ 0 & 0\end{array}\right) \otimes A_{1}$. Similarly the second term can be separated as well. As for the third term, the matrix $B$ is a nonnegative matrix. Suppose $A^{p T}$ has the same row sums as $A$. This implies that the $i$ th column sum of $B$ is equal to its $i$ th row sum. This means that $B$ is line sum symmetric. Using results in network flow theory, it was shown in Ref. [7] that $B$ can be written as $\sum_{i} \alpha_{i} C_{i}$, where $C_{i}$ are simple circuit matrices and $\alpha_{i} \geq 0$. This together with Corollary 1 implies that $A$ is separable in $\mathbb{C}^{2} \otimes \mathbb{C}^{q}$.

Our main result shows that for density matrices in $S_{1}^{0}$, the Peres-Horodecki condition (which by Theorem 3 is equivalent to $A^{p T}$ having zero row sums) is sufficient and necessary for separability in $\mathbb{C}^{2} \otimes \mathbb{C}^{q}$.

Theorem 6 Let $A$ be a $2 q$ by $2 q$ density matrix in $S_{1}^{0}$. The matrix $A$ is separable in $\mathbb{C}^{2} \otimes \mathbb{C}^{q}$ if and only if $A^{p T}$ has zero row sums.

Proof: Follows from Theorems 2 and 5.

\section{A sufficient condition for separability}

The techniques in Section 5 can be applied to density matrices in $S_{1}$ acting on the more general tensor product $\mathbb{C}^{p} \otimes \mathbb{C}^{q}$. In fact, the same arguments can be used to prove the following sufficient condition for separability:

Theorem 7 If an $n$ by $n$ density matrix $A$ in $S_{1}$ written in the form Eq. (1) is such that the matrices $A^{i, j}$ are line sum symmetric, then $A$ is separable in $\mathbb{C}^{p} \otimes \mathbb{C}^{q}$.

Corollary 2 Let $A$ be a density matrix in $S_{1}$ be such that $A_{(i, j)\left(i^{\prime}, j^{\prime}\right)} \neq 0$ implies that $\left|i-i^{\prime}\right| \leq 1{ }^{3}$ If $A^{p T}$ has the same row sums as $A$, then $A$ is separable in $\mathbb{C}^{p} \otimes \mathbb{C}^{q}$.

3 Graphically, if the vertices are arranged as in Fig. 1, then the edges connect vertices in the same row or in adjacent rows. 
Proof: By hypothesis, $A$ is in block tridiagonal form:

$$
A=\left(\begin{array}{cccc}
A^{1,1} & A^{1,2} & \mathbf{0} & \\
A^{2,1} & A^{2,2} & A^{2,3} & \ddots \\
& \ddots & \ddots & \ddots \\
& 0 & A^{p, p-1} & A^{p, p}
\end{array}\right)
$$

If $A^{p T}$ has the same row sums as $A$, then $A^{1,2}$ is line sum symmetric which in turns means that $A^{2,1}=\left(A^{1,2}\right)^{T}$ is line sum symmetric. This implies that $A^{2,3}$ is line sum symmetric etc. The result then follows from Theorem 7.

Corollary 3 Let $A$ be a density matrix in $S_{1}^{0}$ be such that $A_{(i, j)\left(i^{\prime}, j^{\prime}\right)} \neq 0$ implies that $\left|i-i^{\prime}\right| \leq 1$. Then $A$ is separable in $\mathbb{C}^{p} \otimes \mathbb{C}^{q}$ if and only if $A^{p T}$ has zero row sums.

Proof: Follows from Theorem 2 and Corollary 2.

For a normalized Laplacian matrix $A$ of a graph such that $A_{(i, j)\left(i^{\prime}, j^{\prime}\right)} \neq 0$ implies $\left|i-i^{\prime}\right| \leq 1$ and $\left|j-j^{\prime}\right| \leq 1$ (the so-called nearest point graphs), it was shown in Ref. [3] via a combinatorial argument that $A$ is separable if and only if $A^{p T}$ has zero row sums. Corollary 3 is a generalization of this result as the condition $\left|j-j^{\prime}\right| \leq 1$ is not necessary.

\section{Diagonally dominant nonnegative symmetric matrices}

Let $V$ be the set of diagonally dominant nonnegative symmetric matrices, i.e. nonnegative symmetric matrices such that $A_{i i} \geq \sum_{i \neq j} A_{i j}$ for all $i$. By Gershgorin's circle criterion [8] matrices in $V$ are positive semidefinite. Let $V_{1}$ be the set of matrices in $V$ with unit trace. Then $V_{1}$ are density matrices. We obtain results for $V_{1}$ which are analogous to those for $S_{1}$. For instance, we have the following Corollary to Theorem 4:

Corollary 4 If $C$ is a $q$ by $q$ simple circuit matrix and $D$ is the diagonal matrix with the row sums of $C$ on the diagonal, then the matrix

$$
A=\frac{1}{2 \operatorname{Tr}(D)}\left(\begin{array}{cc}
D & C \\
C^{T} & D
\end{array}\right)
$$

is a density matrix and is separable in $\mathbb{C}^{2} \otimes \mathbb{C}^{q}$.

Corollary 4 and the argument in Section 5 are used to prove the following: 
Theorem 8 Let $A$ be a $2 q$ by $2 q$ density matrix in $V_{1}$. If $A^{p T}$ has the same row sums as $A$, then the matrix $A$ is separable in $\mathbb{C}^{2} \otimes \mathbb{C}^{q}$.

Corollary 5 Let $A$ be a density matrix in $V_{1}$ be such that $A_{(i, j)\left(i^{\prime}, j^{\prime}\right)} \neq 0$ implies that $\left|i-i^{\prime}\right| \leq 1$. If $A^{p T}$ has the same row sums as $A$, then $A$ is separable in $\mathbb{C}^{p} \otimes \mathbb{C}^{q}$.

Theorem 9 If an $n$ by $n$ density matrix $A$ in $V_{1}$ written in the form Eq. (1) is such that the matrices $A^{i, j}$ are line sum symmetric, then $A$ is separable in $\mathbb{C}^{p} \otimes \mathbb{C}^{q}$.

\section{Conclusions}

We study separability criteria for density matrices that can be expressed as generalized Laplacian matrices of weighted graphs or diagonally dominant nonnegative matrices. In particular, we show that the well-known PeresHorodecki necessary condition for separability is in fact sufficient for Laplacian matrices in $\mathbb{C}^{2} \otimes \mathbb{C}^{q}$.

\section{References}

[1] M. Nielsen, I. Chuang, Quantum computation and quantum information, Cambridge University Press, 2000.

[2] S. L. Braunstein, S. Ghosh, S. Severini, The laplacian of a graph as a density matrix: a basic combinatorial approach to separability of mixed states, quantph/0406165 (2004).

[3] S. L. Braunstein, S. Ghosh, T. Mansour, S. Severini, R. C. Wilson, Some families of density matrices for which separability is easily tested, quant-ph/0508020 (2005).

[4] A. Peres, Separability criterion for density matrices, Physical Review Letters 77 (8) (1996) 1413-1415.

[5] M. Horodecki, P. Horodecki, R. Horodecki, Separability of mixed states: necessary and sufficient conditions, Physics Letters A 223 (1996) 1-8.

[6] C. Godsil, G. Royle, Algebraic Graph Theory, Graduate Texts in Mathematics, Springer-Verlag, 2001.

[7] G. B. Dantzig, B. C. Eaves, U. G. Rothblum, A decomposition and scalinginequality for line-sum-symmetric nonnegative matrices, SIAM J. Alg. Disc. Meth. 6 (2) (1985) 237-241. 
[8] M. Marcus, H. Minc, A survey of matrix theory and matrix inequalities, Dover, 1992. 